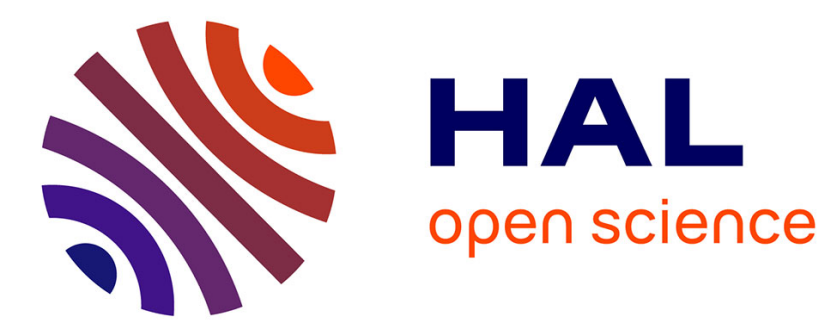

\title{
Model-Driven Safety Assessment of Robotic Systems
}

\author{
Nataliya Yakymets, Saadia Dhouib, Hadi Jaber, Agnes Lanusse
}

\section{To cite this version:}

Nataliya Yakymets, Saadia Dhouib, Hadi Jaber, Agnes Lanusse. Model-Driven Safety Assessment of Robotic Systems. 2013 (IEEE/RSJ) International Conference on Intelligent Robots and Systems, Nov 2013, Tokyo, Japan. cea-01810068

\section{HAL Id: cea-01810068 https://hal-cea.archives-ouvertes.fr/cea-01810068}

Submitted on 7 Jun 2018

HAL is a multi-disciplinary open access archive for the deposit and dissemination of scientific research documents, whether they are published or not. The documents may come from teaching and research institutions in France or abroad, or from public or private research centers.
L'archive ouverte pluridisciplinaire HAL, est destinée au dépôt et à la diffusion de documents scientifiques de niveau recherche, publiés ou non, émanant des établissements d'enseignement et de recherche français ou étrangers, des laboratoires publics ou privés. 


\title{
Model-Driven Safety Assessment of Robotic Systems
}

\author{
N. Yakymets, S. Dhouib, H. Jaber, A. Lanusse
}

\begin{abstract}
Robotic systems (RSs) are often used for performing critical tasks with little or no human intervention. Such RSs must satisfy certain dependability requirements including reliability, availability, security and safety. In this paper, we focus on the safety aspect and propose a methodology and associated framework for safety assessment of RSs in the early phases of development. The methodology relies upon model-driven engineering approach and describes a preliminary safety assessment of safety-critical RSs using fault tree (FT) analysis (FTA). The framework supports a domain specific language for RSs called RobotML and includes facilities (i) to automatically generate or manually construct FTs and perform both qualitative and quantitative FTA, (ii) to make semantic connections with formal verification and FTA tools, (iii) to represent FTA results in the RobotML modeling environment. In the case study, we illustrate the proposed methodology and framework by considering a mobile robot developed in the scope of the Proteus project.
\end{abstract}

\section{INTRODUCTION}

Modern robotic systems (RSs) are complex and capable to perform sophisticated tasks in different domains [1][2]. In order to cope with system complexity, engineers consider new approaches to system development based on formalized modeling [3]. Model-driven engineering (MDE) is expected to significantly simplify the support of system requirements, design, analysis, verification and validation through a system life-cycle. The use of system level models enables simulation of the overall performance and behavior of complex RSs. In addition, MDE gave birth to domain specific languages like Robotic Modeling Language (RobotML) [4] to target system development in different domains. RobotML is built as a UML profile to design, simulate and deploy robotic applications. RSs can be defined using appropriate RobotML notations, abstractions and facilities to automatically generate executable code. RobotML could be a solution for robotics experts to deal with variability problems and to hide the lower level programming details.

N. Yakymets is with the CEA, LIST, DILS, Laboratory of model driven engineering for embedded systems - Point Courrier 174, Gif -sur-Yvette, 91191, France (e-mail: nataliya.yakymets@, cea.fr).

S. Dhouib is with the CEA, LIST, DILS, Laboratory of model driven engineering for embedded systems - Point Courrier 174, Gif-sur-Yvette, 91191, France (e-mail: nataliya.yakymets@cea.fr).

H. Jaber was with the CEA, LIST, DILS, Laboratory of model driven engineering for embedded systems - Point Courrier 174, Gif-sur-Yvette, 91191, France. He is now with the Laboratoire Génie Industriel, Ecole Centrale Paris, Grande voie des vignes, 92290 Chatenay-Malabry, France (e-mail: hadi.jaber@ecp.fr).

A. Lanusse is with the CEA, LIST, DILS, Laboratory of model driven engineering for embedded systems - Point Courrier 174, Gif-sur-Yvette, 91191, France (e-mail: agnes.lanusse@ cea.fr).
Our research has been inspired by RSs for safety-critical applications. Such RSs as [1] or [2] are expected to satisfy a high level of safety. Standards [5][6] concerned with the development of safety-critical systems require an application of specific design flows where system engineering is conducted in parallel with safety assessment (SA) as shown in Figure 1. This allows the concept, design and implementation of safety-critical RS to be developed with respect to the safety aspects. Each phase of SA flow implies application of a set of specific methods and activities. Typical SA methods include preliminary and system hazard and risk analysis [7], fault tree (FT) generation and analysis (FTA) [8], failure mode and effects analysis (FMEA) [9]. Although these well-established methods provide an efficient support for safety engineers, they could greatly benefit from a tighter coupling with system modeling environments. MDE offers facilities to annotate models with information needed for SA, to perform validation according to dedicated rules, to write transformation rules towards formal languages to permit their analysis by formal tools. It becomes thus possible to perform model-driven SA by incorporating existing SA methods and tools into a uniform MDE environment.

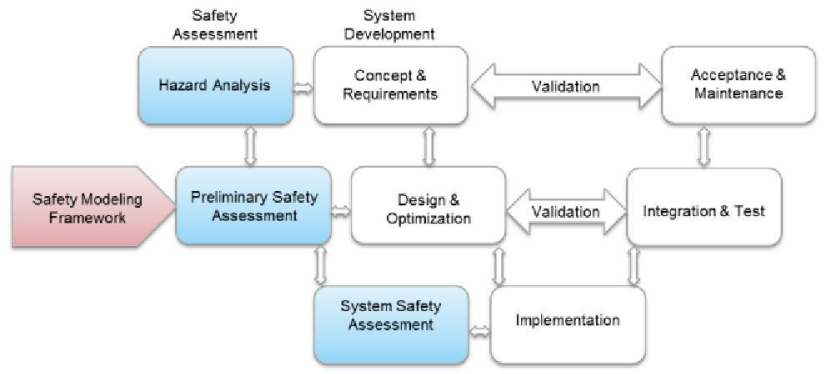

Figure 1. SA-based life-cycle of system development

In this paper, we aim to contribute to integration of SA techniques and standards into the MDE environment based on RobotML. Certain efforts have already been put into investigation of possible ways of SA through the MDE process based on the general purpose System Modeling Language (SysML) [10]. Similar studies are also undertaken with other modeling languages such as architecture description language for automotive embedded systems (EAST-ADL) [11] or architecture analysis and design language (AADL) [12]. However, these languages are limited for RS design compared to the domain specific RobotML language.

We propose a methodology and associated framework for model-driven analysis of RSs in the preliminary SA phase (Figure 1. ). The safety standards for robotics are not mature enough and some of them [13] have been still under development. Therefore the methodology follows the 
IEC61508 [5], a generic standard on functional safety design, and describes SA using FTA approach. It leverages features of RobotML (i) to capture information required for formal analysis (ii) to propagate SA results back into the MDE environment. The framework includes metamodels, profiles, model transformation and FT generation plug-ins, tools for formal verification and FTA. The use of the proposed methodology and framework allows the safety engineer to start SA from the early phases of RS development which can significantly reduce time and cost constraints.

We discuss a case study called Robotic Young Challenge (RYC) which has been designed by the Proteus project partners. RYC addresses problems of autonomous motions of mobile robots in unknown structured environment. Its main functionality is outdoor exploration and target searching. Using this case study, we perform preliminary SA by FT generation and further qualitative and quantitative FTA according to IEC61508. We also show how to use RobotML-based MDE environment to describe possible effects of failures (or dysfunctional behavior) of the Proteus RYC robot and to display generated FTs and FTA results.

The remainder of the paper is organized as follows. In section II, we analyze existing methods and tools for SA. Then we introduce our SA methodology and toolset for RSs in sections III. In section IV, we present the case study on SA and conclude in section V.

\section{RELATED WORKS AND PAPER CONTRIBUTION}

In our research we focus on the preliminary SA phase of SA flow shown in Figure 1. The goals of this phase are (i) to evaluate RS architecture with respect to the list of possible hazards obtained from the hazard analysis phase and (ii) to derive safety requirements. RS architecture can be evaluated using such methods as FTA [8][14][15], FMEA [9][16], event tree analysis, etc. FTA and FMEA are complementary methods aiming to analyze propagation of faults through the system. FMEA is an inductive bottom up method used to analyze a system on component level and check what happens on system level. FTA, a deductive topdown method, does the opposite by defining a state on system level and checking what can cause this at component level. In practice, FTA is performed on larger systems, which makes it more suitable for SA of complex RSs.

FTA was originally developed by H.A. Watson in 1962 at Bell Laboratories [17]. A typical FT consists of the top event and a set of basic and house events organized with the logic gates (AND, OR, etc.). The qualitative analysis of FT aims to find all the minimal combinations of basic events (called minimal cut sets) resulting in the top event. The FT quantitative analysis is also often used in probabilistic computation.

The FT generation approaches fall into several categories depending on the method used to annotate a system with its dysfunctional behavior. Structured

\footnotetext{
${ }^{1}$ http://www.anr-proteus.fr
}

approaches [8][18] use manually created models of failure behavior. Such approaches are time consuming and rely upon the ability of the safety engineer to predict the system behavior and may lead to higher probability of errors. Another group of approaches is based on the decision table method [19]. They are quite efficient for small and middle range systems but may require sophisticated tables for the large systems with complex multi-level hierarchy. Approaches based on failure modes injection extend each component of the nominal system model with a set of possible failure modes and then model the system dysfunctional behavior using such an extended model. The tools based on these approaches (for example, FSAP/NuSMV [20]) translate an extended model into a state machine and then use formal verification algorithms to generate minimal cut sets and construct FTs. In the case of complex systems, however, the application of such approaches may result in combinatorial explosion when the number of failure modes in state machines grows. Some FT generation approaches are based on failure logic modelling. These approaches use analytical expressions associated with the system components to model the possible propagation of failures. HiP-HOPS [21] or SafetyArchitect ${ }^{2}$ tools support failure logic modelling.

TABLE I. METHODS AND TOOLS FOR FTA

\begin{tabular}{|c|c|c|c|c|c|}
\hline SA Tools & Saml & KB3 & $\begin{array}{c}\text { HiP- } \\
\text { Hops }\end{array}$ & $\begin{array}{c}\text { FSAP/Nu } \\
\text { SMV }\end{array}$ & $\begin{array}{c}\text { Our MSA } \\
\text { framework }\end{array}$ \\
\hline $\begin{array}{c}\text { FT } \\
\text { generation } \\
\text { method }\end{array}$ & $\begin{array}{l}\text { Min. } \\
\text { cut } \\
\text { sets }\end{array}$ & $\begin{array}{c}\text { Ma- } \\
\text { nual }\end{array}$ & $\begin{array}{c}\text { Analy- } \\
\text { tical } \\
\text { expr. }\end{array}$ & $\begin{array}{c}\text { Min. cut } \\
\text { sets }\end{array}$ & $\begin{array}{c}\text { Analytical } \\
\text { expr., Min. } \\
\text { cut sets }\end{array}$ \\
\hline $\begin{array}{c}\text { Support of } \\
\text { hierarchical } \\
\text { systems }\end{array}$ & $\checkmark$ & - & $\checkmark$ & $\checkmark$ & $\checkmark$ \\
\hline FTA & $\checkmark$ & $\checkmark$ & $\checkmark$ & $\checkmark$ & $\checkmark$ \\
\hline $\begin{array}{c}\text { Formal } \\
\text { verification }\end{array}$ & $\checkmark$ & - & - & $\checkmark$ & $\checkmark$ \\
\hline $\begin{array}{c}\text { Input } \\
\text { language }\end{array}$ & Saml & $\begin{array}{c}\text { Figa- } \\
\text { ro }\end{array}$ & $\begin{array}{c}\text { Matlab } \\
\text { EAST- } \\
\text { ADL }\end{array}$ & SMV & $\begin{array}{c}\text { RobotML, } \\
\text { AltaRica, } \\
\text { OpenPSA }\end{array}$ \\
\hline
\end{tabular}

The comparative analysis of existing approaches and tools for FT generation and analysis is given in TABLE I. We list here only academic approaches, since industrial solutions generally rely on a part of them. Although some of these tools [20][21] perform automatic FT generation, their capabilities are limited for SA of complex RSs. First, they lack convenient representation of the input system models. For example, FSAP/NuSMV, SAML [22] or $\mathrm{ARC}^{3}$ tools use formal symbolic languages such as SMV, SAML [22] or AltaRica [23] to describe a system. This might require certain time efforts from the SA engineer to formulate and enter the model in these formats. Second, they lack a convenient representation of the final results of SA. In HiPHOPS, for instance, safety annotations can be entered through a profile of the EAST-ADL implementation in the Papyrus ${ }^{4}$ tool, but there are no elaborated mechanisms to

\footnotetext{
${ }^{2} \mathrm{http} / / / \mathrm{all}$ tec.net/index.php/en/model-based-safety-analysis/25-safetyarchitect-a-mbsa-tool

${ }^{3} \mathrm{http} / / /$ altarica.labri.fr/forge/projects/arc/wiki

${ }^{4}$ http://www.eclipse.org/modeling/mdt/papyrus/
} 
show the results of conducted SA in the system modeling environment.

In this work, we analyze the possibilities of using different methods and tools for model-driven SA during the early phases of RS development. The IEC61508 standard [5] refers to FTA as a recommended technique to perform $\mathrm{SA}$ in the design phase of system life-cycle. We propose a methodology to perform a preliminary SA of RSs using FTA and then to derive the obtained results back into the RobotML model. To automatically generate FTs from RobotML models, we combine the approach based on analytical expression of dysfunctional behavior with formal verification based on AltaRica language. The AltaRica model is automatically generated from the RobotML annotated model using transformation rules. The methodology was implemented in a framework which integrates formal verification and FTA algorithms in the MDE environment supported by the Papyrus editing tool for RobotML. The framework contains model transformation and FT generation plug-ins, as well as profiles for model annotation and FT visualization. The qualitative and quantitative FTA is carried out with built-in ARC and $\mathrm{XFTA}^{5}$ engines.

In the next sections, we shall describe the methodology and framework, and show how they can be used for SA of RSs.

\section{METHODOLOGY AND FRAMEWORK}

The methodology is dedicated to the preliminary SA of safety-critical RSs. Figure 2. illustrates the SA flow based on the use of the proposed methodology. The following information is taken as input data:

- the $S A$ recommendations taken from the IEC61508 standard;

- the list of possible hazards derived from the hazard analysis phase of SA flow;

- the system architecture defined as a multi-level network of RobotML components like Sensors, Actuators, etc.

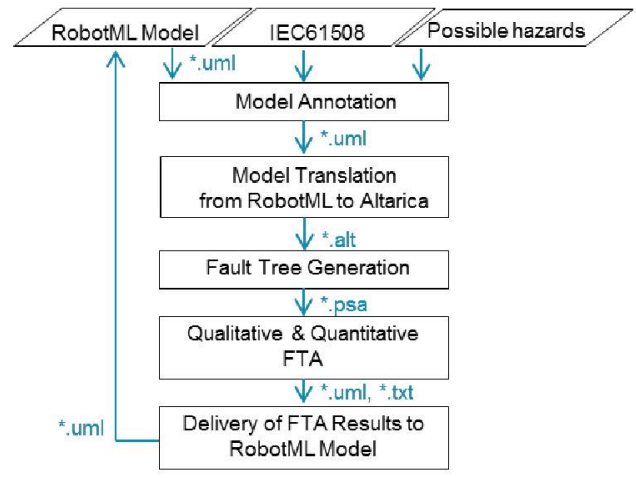

Figure 2. The SA methodology
First, a RS is designed with the Papyrus platform using RobotML language. Second, we define the sufficient finest level of RS architecture where SA will be conducted and then annotate a RobotML model with the possible failure behavior at this level. While defining failure modes of the components, information on the possible hazards derived from the hazard analysis is taken into account. RS dysfunctional behavior is annotated using analytical expressions. Once the annotation has been done, the failure states and events related to the component failure modes are automatically extracted, and the RobotML model is converted into the AltaRica language. The checking of the AltaRica model is performed by the ARC tool. This tool also computes minimal cut sets for the considered model. Based on this information we automatically generate FTs and represent them in the Open-PSA format ${ }^{6}$. Finally, we perform FT qualitative and quantitative analysis according to IEC61508 and compute a set of factors (like probability of the top FT event, contribution of minimal cut sets, etc.) to evaluate system safety. In order to make SA results more representative, we display FTs in RobotML modeling environment with FT profile.

The complexity of the proposed methodology is strongly linked to the number of levels in the system hierarchy and to the scalability of the formal verification tool used. If the number of potential failure modes increases, the risk of combinatorial explosion is higher. The proposed methodology provides a possibility to control the granularity of SA through the assessment process by choosing the finest level of RS architecture where the components are annotated with the dysfunctional behavior. Therefore it is a flexible instrument to control the number of failure modes and to decrease the risk of combinatorial explosion.

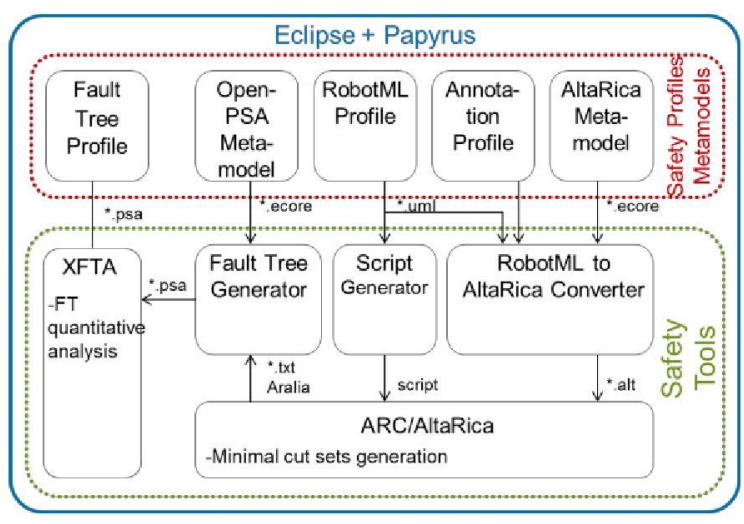

Figure 3. The architecture of the framework

The architecture of our framework is represented in Figure 3. It has been implemented using java under Eclipse Modeling Framework (EMF). The framework contains two sets of tools including safety profiles and metamodels and tools for SA at the preliminary SA phase including FT generation and analysis.

In order to illustrate the proposed SA methodology and framework as clearly as possible, we consider the example of

\footnotetext{
${ }^{5} \mathrm{http}: / /$ www.lix.polytechnique.fr/ -rauzy/xfta/xfta.htm
}

${ }^{6} \mathrm{http}: / /$ www.open-psa.org 
a mobile robot and go through the safety modeling flow associated with our approach in the next section.

\section{CASE STUdy}

We validate the methodology and framework described in the previous sections by analyzing one of the case studies developed in the scope of the Proteus project. The considered example is a scenario defined by Robotic Youth Challenge (RYC) and deployed in a WifiBot robot ${ }^{7}$. The RYC targets autonomous motion of mobile robots in unknown structured environment. The main scenario for the robot is outdoor exploration and target searching. The RYC architecture developed in RobotML using RobotML modeling environment is shown in Figure 4. The top hierarchical level includes nine components: Mission Generator generates RYC missions, Path Planner calculates a path for the RYC robot using the global map and information on current mission and position, Navigator delivers local trajectory for the pilot taking into account the local map, Pilot calculates the left and right wheel speed setpoints that the robot is supposed to reach to follow the input trajectory, Servoings transforms the speed commands to the format (tics) used by the wifibot robot, Sensors captures information on surrounding environment, Proximetry builds a map in polar coordinate with only meausres from sensors directly printed in it, Local Map builds a relative Cartesian 2D map with obstacles placed in it, Global Map builds an absolute map of the scene.

In this example, we consider a hazardous event when "The RYC robot does not follow the commands". In other words, this will be a top event of the tree.

\section{A. Model Annotation}

The RYC model described in RobotML is annotated with failure behavior. Information on hazards, derived from the hazard analysis according IEC61508, is taken into account while defining possible failures of RYC components. The dysfunctional behavior is represented as a set of analytical expressions showing how deviations in the component outputs can be caused by internal failures of the component and/or possible deviations in the component inputs. Only components of the finest level defined for SA (or basic components, BCs) are annotated with the analytical expressions. For example, the output deviation expression for the output Path of the BC called Path Planner (Figure 4. ) has the following format:

\section{(NOTf) AND Mission_Type AND Position AND Map.}

It means that the output Path does not propagate failure behavior if (i) there is no failure $f=$ "Path Planner internal failure" of the component Path Planner and (ii) information on the input ports Mission_Type, Position and Map is correct.

The dysfunctional behavior of the components representing higher hierarchical levels is simulated by model checking engine. It is a composition of state machines obtained after model transformation into the

\footnotetext{
${ }^{7}$ http://www.wifibot.com
}

AltaRica language from the output deviation expressions of BCs.

We assign output deviation expressions using a UML profile mechanism in Papyrus environment. The framework contains an annotation profile enabling to stereotype each $\mathrm{BC}$ output port with deviation expressions. During the model translation process, the failure states and events related to the RYC components are automatically extracted from these expressions.

\section{B. Model Translation}

The next step is to extract information on the failure states and events from the output deviation expressions and to convert the RobotML model into the AltaRica language. The transformation method used for conversion of RobotML model to AltaRica language relies upon the MDE approach. TABLE II. lists the mapping we defined for our transformation algorithm implemented in the framework.

TABLE II. TRANSFORMATION RULES

\begin{tabular}{|l|l|l|l|}
\hline Concept & RobotML & AltaRica & Descr. \\
\hline $\begin{array}{l}\text { Component } \\
\text { type }\end{array}$ & Robot & Node main & $\begin{array}{l}\text { RS under } \\
\text { analysis }\end{array}$ \\
\hline Component & $\begin{array}{l}\text { Software, Hardware, RoboticSysten, } \\
\text { SensorSystem, ActuatorSystem, } \\
\text { CameraSystem, GPSSystem, Object- } \\
\text { DetectionSensorSystem, Sensor- } \\
\text { Driver, ImageSensorSystem, } \\
\text { EngineSystem, WheelSystem, } \\
\text { ObjectTrackingSensorSystem, } \\
\text { LocalizationSensorSystem, } \\
\text { SimulatedSystem } \\
\text { Part }\end{array}$ & Node & $\begin{array}{l}\text { RS } \\
\text { components }\end{array}$ \\
/Prototype & $\begin{array}{l}\text { DataFlowPort } \\
\text { /Type }\end{array}$ & Field:sub & \\
\hline $\begin{array}{l}\text { Flow variable } \\
\text { /Type }\end{array}$ & / Direction & $\begin{array}{l}\text { Field: Flow } \\
\text { /bool,integer, } \\
\text { float,domain } \\
\text { /In, Out }\end{array}$ & RS ports \\
\hline $\begin{array}{l}\text { Connection } \\
\text { components }\end{array}$ & Connector & Assertion & $\begin{array}{l}\text { Connection } \\
\text { between } \\
\text { components }\end{array}$ \\
\hline $\begin{array}{l}\text { Ontput } \\
\text { deviation } \\
\text { expression }\end{array}$ & Stereotyped DataFlowPort & $\begin{array}{l}\text { Failure states and } \\
\text { events, output } \\
\text { assertions }\end{array}$ & $\begin{array}{l}\text { Component } \\
\text { failure } \\
\text { behavior }\end{array}$ \\
\hline
\end{tabular}

By default, we assume that the RYC robot is operating normally. Consequently, all extracted failure states associated with BCs (or nodes in AltaRica) are initialized as "false" in AltaRica. Based on information on the extracted failure states, we create a set of events resulting in the occurrence of these states and then generate appropriate transactions. The declaration of the main node in AltaRica model relies upon information extracted from RobotML top architecture diagram: the system parts are translated into sub-nodes connected via assertions

\section{Fault Tree Generation and Analysis}

In this phase RYC is assessed by using FTA method. The framework uses integrated model checking engine called ARC and script generator to compute minimal cut sets for a considered top event. Then FT is built with FT generator. We consider only static FTs, however, the ARC engine can provide the facilities to further analyze a dynamic behavior of RSs.

FT generation includes several steps. First, we obtain all possible minimal combinations of component failures violating a given failure event. Second, we group these combinations, called minimal cut sets, in a tree structure as 
follows. The events from each minimal cut set are considered as basic and grouped using AND gate. Then we connect all the AND gates to the OR gate which, in turn, is linked to the top event.

The qualitative FTA has shown that the top event "The RYC robot does not follow the commands" occurs if any sequence of basic failure events given in TABLE III. occurs. Once a FT has been obtained, we carry out a quantitative FTA using integrated XFTA engine. This engine performs quantitative analysis of FTs and provides information on the top event probability for different mission times, importance factors of basic events, common cause failure analysis, etc. According to the standard IEC61508, we assess the probability of the considered top event based on the statistical data on failure rates of basic events of the considered components. In addition, the probability and contribution of each minimal cut set are computed (TABLE III. ). Moreover, we define the most critical part of RYC, the Sensors sub-system, since its failure has the highest impact on the failure of the whole RS.

\section{Propagation of FTA Results}

The automatically generated FT can be either represented in open-PSA format, the FT specific format developed for describing complex FTs, or in a graphical form via dedicated profile. By using the FT profile, we can present FTs that consist of basic, house and top events organized with AND or OR gates, as well as FTA results. Thus, the use of such a profile helps to construct FTs in RobotML/Papyrus environment and provides a better connection between system functional and dysfunctional behavior through MDE.

\section{CONClusion}

In this work, we propose the methodology and framework which provide a support for safety engineers by integrating safety techniques within a model-driven engineering process. The methodology relies on the generic standard on functional safety design IEC61508 and shows how to automate safety assessment process of robotic systems in the early development phases. The use of the proposed methodology aims to fill the gap between system modeling and safety assessment tools and helps to better cope with system engineering time and cost constraints. Indeed, the results of preliminary safety assessment can reveal the most safety-critical parts of the system which should be mitigated.

To implement the proposed methodology, we develop a safety modeling framework which automates safety assessment of robotic applications in the RobotML-based modeling environment. The framework is an alternative to such safety assessment tools as HiP-HOPS, FSAP/NuSMV, KB3, SAML. As opposed to these tools, the framework is oriented to the robotic domain and provides the facilities of RobotML domain specific language to develop safetycritical robotic applications. Furthermore, the framework supports a common system model for system and safety engineers, by using UML profile mechanisms in Papyrus. This allows to integrate all data linked with safety assessment in the same system model, as well as to customize an interface to show different results within one uniform environment and reuse this information for further reliability studies.

\section{ACKNOWLEDGMENT}

The authors thank Hadi Jaber for his strong contribution to this work during his internship in CEA.

\section{REFERENCES}

[1] J.J. Biesiadecki, M.W. Maimone, "The Mars exploration rover surface mobility flight software: driving ambition," IEEE Aerospace Conference Proceedings, March 2006, Montana, USA.

[2] A. Rankin, C. Bergh, S. Goldberg, and L. Matthies, "Passive perception system for day/night autonomous offroad navigation," In SPIE UGV Symposium, Orlando, FL, April 2005.

[3] J. A. Estefan, "Survey of model-based Systemse (MBSE) methodologies," Rev A, Incose MBSE Focus Group, 2007.

[4] S. Dhouib, S. Kchir, S. Stinckwich, T. Ziadi, M. Ziane, "RobotML, a domain-specific language to design, simulate and deploy robotic applications," Int. Conference on Simulation, Modeling and Programming for Autonomous Robots, pp. 149-160, 2012.

[5] IEC, 61508: 1998 and 2000, part 1 to 7. Functional safety of electrical, electronic and programmable electronic systems, 2000.

[6] ARP-4754: Certification considerations for highly-integrated or complex aircraft systems, Society of Automotive Engineers (SAE) standard, 1996.

[7] IEC 61882: Hazard and operability studies, application guide, 2001.

[8] Fault tree handbook with aerospace applications. NASA, 2002.

[9] IEC 60812: Analysis techniques for system reliability - Procedures for FMEA, 1985.

[10] P. David et al., "Reliability study of complex physical systems using SysML," Reliability Engineering and system Safety, Elsevier, pp. 431-450, 2010.

[11] P. Cuenot, D.J. Chen, S. Gerard, H. et al. Towards improving dependability of automotive systems by using the EAST-ADL architecture description language. Architecting Dependable Systems IV, Lecture Notes in Computer Science, vol. 4615, pp, 39-65, 2006.

[12] P. H. Feiler, D. P. Gluch, "Model-based engineering with AADL: an introduction to the SAE architecture analysis \& design language," Addison-Wesley Professional, 1st ed., pp. 496, 2012.

[13] ISO/FDIS13482: Robots and robotic devices - Safety requirements for non-industrial robots - Non-medical personal care robots (Final Draft International Standard).

[14] C. Carreras, I.D. Walker, "Interval methods for fault-tree analysis in robotics," IEEE Trans. on Reliability, Vol. 50, No. 1, 2001, pp. 3-11.

[15] I.D. Walker, J.R. Cavallaro, "Failure mode analysis for a hazardous clean-up manipulator," Reliability Engineering and System Safety, Special Issue on Safety of Robotic Systems, Vol. 53, No3, September 1996, pp. 277-290

[16] M.L. Visinsky, J.R. Cavallaro and I.D. Walker, "Robotic fault detection and fault tolerance: a survey," Reliability Engineering and System Safety, Vol. 46, \#2, 1994, pp. 139-158.

[17] C. Ericson, "Fault Tree Analysis - A History," Proceedings of the 17th International Systems Safety Conference, 1999.

[18] I. Renault, et al.,"KB3: computer program for automatic generation of fault trees," Rel. Maintain. Symp., pp. 389 -395, 1999

[19] J. D. Andrews, J. J. Henry, "A computerized fault tree construction methodology," in Proc. of the Institution of Mechanical Engineers, 1997; 211(E), pp. 171-183.

[20] M. Bozzano, Ch. Jochim, "The FSAP/NuSMV-SA safety analysis platform," Int. Journal on Software Tools for Technology Transfer, 2007,v.9, №1, pp. 5-24.

[21] M. Walker et al., "Compositional temporal fault tree analysis. Computer safety, reliability, and security," Lecture Notes in Computer Science, vol. 4680, 2007, pp. 106-119. 
[22] M. Gudemann, F. Ortmeier, "A Framework for qualitative and quantitative formal model-based safety analysis," In Proc. 12th IEEE High Assurance Systems Engineering Symp., pp. 132-141, 2010.

[23] A. Arnold, A. Griffault, G. Point, A. Rauzy, "The AltaRica language and its semantics," In Fundamenta Informaticae, vol. 34, pp 109 124,2000

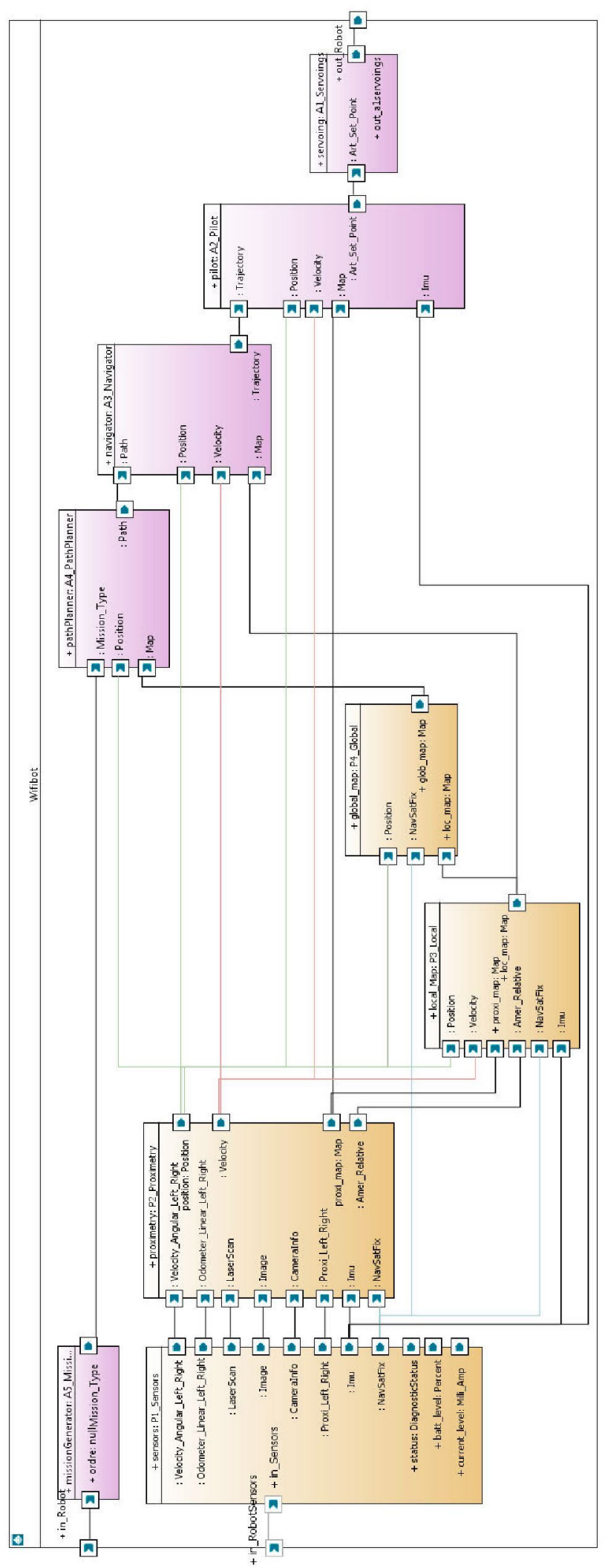

Figure 4. The RYC architecture
TABLE III. FAULT TREE ANALYSIS ReSUlts

\begin{tabular}{|c|c|c|c|}
\hline \multicolumn{2}{|c|}{ Qualitative FTA } & \multicolumn{2}{|c|}{ Quantitative FTA } \\
\hline $\mathbf{N}$ & Minimal Cut Set & Probability & Contribution \\
\hline 1 & $\begin{array}{l}\text { (sensors.camera_FireWire.Incorrect_video_c } \\
\text { apturing_occurs, } \\
\text { sensors.camera_FireWire.Incorrect_calibratio } \\
\text { n_occurs) }\end{array}$ & 0.0000045 & 0.0000370239 \\
\hline 2 & $\begin{array}{l}\text { (sensors.camera_FireWire.internal_Camera_f } \\
\text { ailure_occurs) }\end{array}$ & 0.008 & 0.0658203 \\
\hline 3 & $\begin{array}{l}\text { (sensors.LaserRange.Incomect_laser_scan_oc } \\
\text { curs) }\end{array}$ & 0.004 & 0.0329102 \\
\hline 4 & $\begin{array}{l}\text { (pilot.pilot_Pilot_doesnt_avoid_obstacles_see } \\
\text { n_in_approximetric_map_occurs) }\end{array}$ & 0.0065 & 0.053479 \\
\hline 5 & $\begin{array}{l}\text { (pilot.pilot.Pilot_follows_wrong_trajectory_ } \\
\text { when_calculating_set_points_in_operating_sp } \\
\text { ace_occurs) }\end{array}$ & 0.003 & 0.0246826 \\
\hline 6 & $\begin{array}{l}\text { (pilot.iKM.IKM_doesnt_transform_velocities } \\
\text { from_operational_to_articular_space_occurs }\end{array}$ & 0.002 & 0.0164551 \\
\hline 7 & $\begin{array}{l}\text { (navigator.navigator.Incorrect_velocity_analy } \\
\text { sis_occurs, } \\
\text { navigator.navigator.Incorrect_position_analys } \\
\text { is_occurs, } \\
\text { navigator.navigator.Incorrect_local_map_ana } \\
\text { lysis_occurs, } \\
\text { navigator.navigator.Incorrect_path_analysis_ } \\
\text { occurs) }\end{array}$ & 0.0015 & 0.0123413 \\
\hline 8 & $\begin{array}{l}\text { (navigator.navigator.Internal_failure_of_Navi } \\
\text { gator_occurs) }\end{array}$ & 0.005 & 0.0411377 \\
\hline 9 & $\begin{array}{l}\text { (missionGenerator.mission.internalFailure_oc } \\
\text { curs) }\end{array}$ & 0.002 & 0.0164551 \\
\hline 10 & $\begin{array}{l}\text { (sensors.wifibot_Frame_Out.internalFailure } \\
\text { occurs) }\end{array}$ & 0.003 & 0.0246826 \\
\hline 11 & (sensors.odometer.internalFailure_occurs) & 0.0015 & 0.0123413 \\
\hline 12 & $\begin{array}{l}\text { (proximetry.amer_Identif.Incorrect_interpreta } \\
\text { tion_camera_results_occurs) }\end{array}$ & 0.005 & 0.0411377 \\
\hline 13 & $\begin{array}{l}\text { (proximetry.amer_Identif.Internal_failure_Am } \\
\text { er_Identif_occurs) }\end{array}$ & 0.003 & 0.0246826 \\
\hline 14 & $\begin{array}{l}\text { (proximetry.proximetric_Map.Incontect_bit_ } \\
\text { map_generation_occurs) }\end{array}$ & 0.0065 & 0.053479 \\
\hline 15 & $\begin{array}{l}\text { (proximetry_proximetric_Map.Internal_failure } \\
\text { _Proximetric_Map_occurs) }\end{array}$ & 0.006 & 0.0493652 \\
\hline 16 & (sensors.IMU.internalFailure_occurs) & 0.001 & 0.00822754 \\
\hline 17 & $\begin{array}{l}\text { (proximetry.superDKM.internalFailure_occur } \\
\text { s) }\end{array}$ & 0.007 & 0.0575928 \\
\hline 18 & $\begin{array}{l}\text { (pathPlanner.path_Planner. Wrong path_gene } \\
\text { ration based on correct input_data_occurs) }\end{array}$ & 0.003 & 0.0246826 \\
\hline 19 & $\begin{array}{l}\text { (pathPlanner.path_Planner.Intemal_failure_of } \\
\text { Path_Planner_occurs) }\end{array}$ & 0.0065 & 0.053479 \\
\hline 20 & $\begin{array}{l}\text { (proximetry.superDKM.Wrong_velocity_calc } \\
\text { ulation_using_correct_input_data_occurs) }\end{array}$ & 0.003 & 0.0246826 \\
\hline 21 & $\begin{array}{l}\text { (proximetry.superDKM.Wrong_position_calc } \\
\text { ulation_using_correct_input_data_occurs) }\end{array}$ & 0.0035 & 0.0287964 \\
\hline 22 & $\begin{array}{l}\text { (proximetry.superDKM.Internal_failure_Supe } \\
\text { r_DKM_module_occurs) }\end{array}$ & 0.006 & 0.0493652 \\
\hline 23 & $\begin{array}{l}\text { (local_Map.amer_Loc.Internal_failure_Amer } \\
\text { Relative_occurs) }\end{array}$ & 0.005 & 0.0411377 \\
\hline 24 & $\begin{array}{l}\text { (local_Map.local_Map.Incorrect_analysis_of_ } \\
\text { proximetric_map_occurs, } \\
\text { local_Map.local_Map.Incorrect_analysis_of_ } \\
\text { GPS_data_occurs, } \\
\text { local_Map.local_Map.Incorrect_analysis_ofv } \\
\text { elocity_occurs, } \\
\text { local_Map.local_Map.Incorrect_analysis_of_ } \\
\text { position_occurs) }\end{array}$ & 0.0000157 & 0.00011565 \\
\hline 25 & $\begin{array}{l}\text { (local_Map.local_Map.Memory_failure_occu } \\
\text { Is) }\end{array}$ & 0.007 & 0.0575928 \\
\hline 26 & $\begin{array}{l}\text { (local_Map.local_Map.Internal_failure_Local } \\
\text { _Map_occurs) }\end{array}$ & 0.0015 & 0.0123413 \\
\hline 27 & $\begin{array}{l}\text { (global_map.global_Map.Incorrect_analysis_ } \\
\text { of_position_data_occurs, } \\
\text { global_map_global_Map.Incorrect_analysis_o } \\
\text { f_local_map_data_occurs, } \\
\text { global_map.global_Map.Incorrect_analysis_o } \\
\text { f_GPS_data_occurs) }\end{array}$ & 0.0000167 & 0.00005877 \\
\hline 28 & $\begin{array}{l}\text { (global_map.global_Map.Internal_failure_of_- } \\
\text { GlobalMap_module_occurs) }\end{array}$ & 0.004 & 0.0329102 \\
\hline 29 & (sensors.GPS.Internal_GPS_failure_occurs) & 0.008 & 0.0658203 \\
\hline 30 & (in_Robot._isAbsent) & 0.005 & 0.0411377 \\
\hline 31 & $\begin{array}{l}\text { (servoing.servoings.Incorrect_interpretation } \\
\text { of_command_Left_occurs, } \\
\text { servoing.servoings.Incorrect_interpretation_o } \\
\text { f_command_Right_occurs) }\end{array}$ & 0.0000385 & 0.00031676 \\
\hline 32 & $\begin{array}{l}\text { (servoing.frame_In.Internal_failure_of_modul } \\
\text { e_WifiBot_Frame_In_occurs) }\end{array}$ & 0.0045 & 0.0370239 \\
\hline \multicolumn{2}{|c|}{ Top Event } & \multicolumn{2}{|l|}{ Probability } \\
\hline \multicolumn{2}{|c|}{ The RYC robot does not follow the commands } & 0.121543 & \\
\hline
\end{tabular}

